\title{
Locating Direction Finders Optimally under Risk of Detection
}

\author{
Suhwan Kim * \\ Department of Military Science, Korea National Defense University, Republic of Korea
}

\begin{abstract}
The military uses direction finders (DFs) to determine the location of enemy forces by estimating the positions of their transmitters, which emit radio frequencies. This paper considers the problem of locating DFs with the goal of maximizing the accuracy with which transmitter positions can be estimated in a target area while managing the expected number of DFs that will not be detected by the enemy. Once detected, a DF is subject to jamming or attack by the enemy. This paper presents six models, each appropriate for a different battlefield situation. It casts three models as network flow problems and presents an efficient label-setting algorithm to solve them. The remaining formulations represent novel applications of the Conditional Value at Risk (CVaR) to deal with the probability of DF detection. Computational tests compare model solutions.
\end{abstract}

Keywords Direction Finder, Military Application, Risk Management, Conditional Value at Risk, Label-setting Algorithm

\section{AMS 2010 subject classifications 90B 80}

DOI: $10.19139 /$ soic.v6i2.399

\section{Introduction}

A direction finder (DF) can estimate the direction from which a radio wave arrives at a location. This capability finds many applications, including locating downed aircraft or disabled ships. In the military, a DF system with multiple DFs can be used to identify the locale of enemy forces by estimating the position from which an enemy transmitter emits radio frequencies (for a description of how a DF system operates, see [9]). It is important to estimate enemy positions as accurately as possible while locating DFs to avoid detection by the enemy.

The research objectives of this study are threefold. The first is to formulate six models to locate DFs under diverse battlefield situations, each of which deals explicitly with the probability that the enemy will detect a DF in a different way. The second is to present an efficient label-setting algorithm to solve the models, each cast as a constrained shortest-path problem with one equality and one greater-than-or-equal-to inequality. The third objective is to perform computational tests to compare solutions prescribed by our formulations, to identify tradeoffs between the accuracy with which enemy transmitter positions can be estimated and the likelihood that the enemy will detect DF locations, and to reveal insights that commanders can use as guidelines.

This work is motivated by the critical role that DFs play in the modern, electronic battlefield. The U.S. Army field manual, FM 34-40-9 [1], which prescribes the use of DFs, emphatically states the need to assure that they are located in such a manner as to avoid detection by the enemy. If the enemy is able to detect the location of a DF, it may degrade DF capabilities by electronically jamming it or seek to destroy it, for example, by attacking it using special forces, aircraft or artillery. Some prior research has proposed methods to locate DFs to maximize the detection of enemy transmitters, but none has considered the possibility of DFs being detected by the enemy. This paper, therefore, addresses a troublesome deficiency.

\footnotetext{
*Correspondence to: Suhwan Kim (Email: ksuhwan@kndu.ac.kr). Department of Military Science, Korea National Defense University. 1040 Hwangsanbeol-ro, Yangchon-myon, Nonsan-si, Chungcheongnam-do, Republic of Korea (33021).
}

ISSN 2310-5070 (online) ISSN 2311-004X (print)

Copyright (C) 2018 International Academic Press 
Throughout this paper, we associate the term location uniquely with a DF and the term position uniquely with an enemy transmitter. We assume that the DF system operator can identify potential locations for DFs; our models prescribe a subset of them to locate DFs. We borrow several terms from U.S. Army field manuals FM 34-40-9 and FM 101-5-1 [1,2]. The forward line of own troops (FLOT) indicates the locus of the most forward friendly forces in an Army operation. The expected target area (ETA) designates the area occupied by enemy forces. We use the term locale to designate where enemy forces are within the ETA. DFs can be located on the side of the FLOT controlled by friendly forces. Upon detecting an emission, a DF indicates the approximate direction, commonly called a line of bearing (LOB), in which the transmitter $(t)$ lies. Figure 1 depicts an example with the FLOT separating the ETA and its likely transmitter positions from the area controlled by friendly forces, which includes potential DF locations, the true $L O B_{i}$ from DF location $\ell_{i}$ to actual transmitter position $t$, and a $L O B_{i}$ that might actually be reported by the DF at $\ell_{i}$.

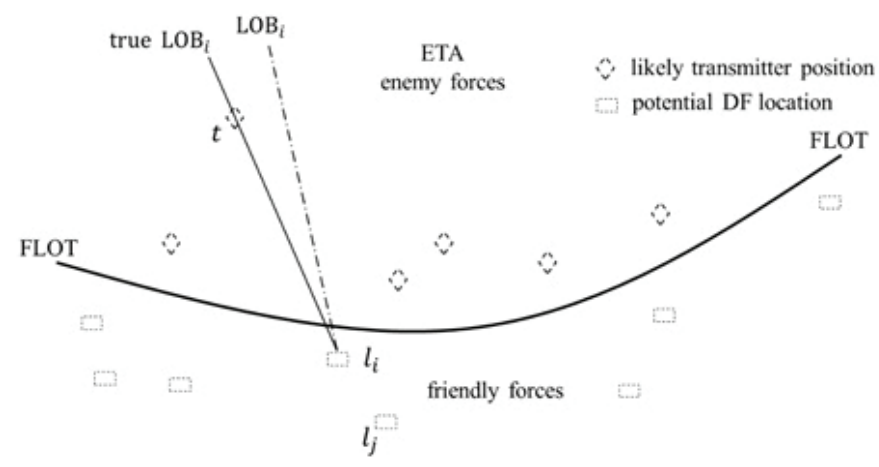

Figure 1. Conceptual depiction of a battlefield

Research on locating DFs is meager. Kennedy and Woolsey [6] suggested empirical strategies, recommending that each pair of DFs be separated by at least the distance between the centroids of the ETA and of the DF locations and that the locations of three or more DFs should not be co-linear. Lee et al. [10] contributed the first quantitative method, a heuristic called the line method, which is based on simulation to maximize a measure of performance they called the probability of coverage (POC) of likely transmitter positions. They defined POC as the probability that the position $t^{\prime}$ estimated by the line method will be within distance $\mathrm{r}$ of actual transmitter position $t$.

Lee et al. [10] and Kim and Wilhelm [8] argued that the accuracy with which a transmitter position is estimated is highly dependent upon the locations of DFs relative to the position of a transmitter. That is, the DF system operator must locate DFs so that the positions of transmitters within the ETA can be most accurately estimated. In this study, we focus on locating DFs with the goal of accurately estimating the positions of enemy radio transmitters, while managing the expected number of DFs that will not be detected by the enemy.

The body of this paper is organized in four sections. Section 2 briefly explains the deterministic version of the DF deployment problem studied by Kim and Wilhelm [8]. Section 3 presents our six models of the DF location problem and the solution methods we propose. Section 4 presents our computational tests and compares solutions prescribed by our models. Finally, section 5 relates our conclusions and outlines fertile directions for future research.

\section{Deterministic DF deployment problem}

So that this paper is self-contained for reader convenience, we begin by describing the deterministic version of the DF location problem. Kim and Wilhelm [8] proposed three methods that optimize a surrogate measure for POC (see Lee et al, [10]) that is easier to compute. We briefly introduce one of the Kim-and-Wilhelm methods, which we extend in this paper (see Kim and Wilhelm [8] for details). We explain how a DF system operates, supposing a transmitter is positioned at $t$ and DFs are deployed at locations $l_{1}, l_{2}$, and $l_{3}$, numbered left to right, as shown in Figure 2. Upon detecting an emission, $D F i$ indicates the approximate direction, $L O B_{i}(i=1,2,3)$. The $L O B_{i}$ 
includes an angular error $\left(\epsilon_{i}\right)$ that is typically assumed to follow a normal distribution with mean 0 and standard deviation $\sigma_{i}(i=1,2,3)$ (Lee et al, [10], Stansfield [13]).

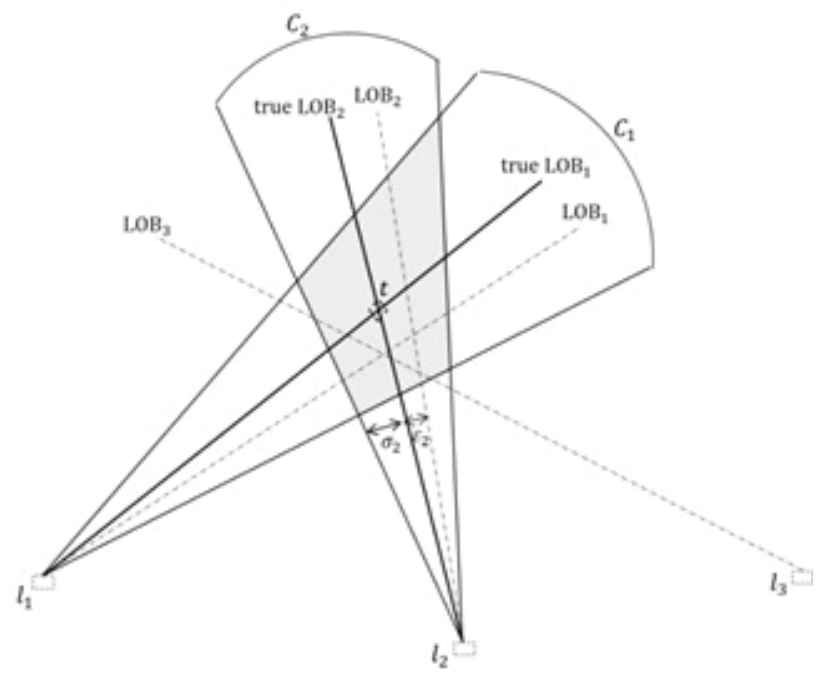

Figure 2. Error cones $C_{1}$ and $C_{2}$ formed by $L O B_{1} \pm \sigma_{1}$ and $L O B_{2} \pm \sigma_{2}$

Under the assumption that angular measurement error is normally distributed, the true $L O B_{i}$ plus and minus one $\sigma_{i}$ (i.e., $L O B_{i} \pm 1 \sigma_{i}$ ) forms an error cone $C_{i}$ that includes $68.2 \%$ of the bearing estimates that $\mathrm{DF} i$ would report. Kim and Wilhelm [8] used the area of a quadrilateral formed by the intersection of the error cones generated by two DFs as their surrogate measure and prescribed DF locations to minimize the sum of the surrogate measures associated with adjacent pairs (i.e., $l_{1}$ and $l_{2}, l_{2}$ and $l_{3}$ ) of DFs.

By exploiting these surrogate measures, they cast the DF location problem as a resource constrained shortestpath problem (CSPP) on the network in Figure 3, in which each node (except start and end nodes) represents a potential DF location; and each arc, the selection of a pair of locations. Figure 3 illustrates such a network with $m=6$ potential DF locations; nodes s and e are dummy start and end nodes, respectively.

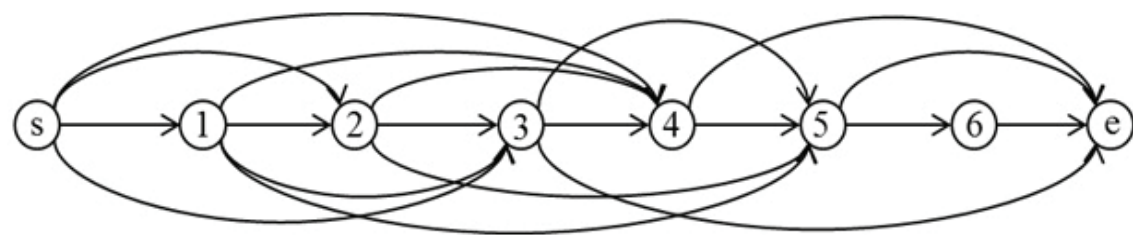

Figure 3. Network for DF location problem

A feasible path with $n+2$ nodes, including start and end nodes, prescribes the locations of $\mathrm{n}$ DFs. Based on this network, Kim and Wilhelm [8] formulated the DF location problem as a CSPP, using the following notation:

Indices

$i, j$ nodes: $i<j$

$s$ start node

$e$ end node

$t$ likely transmitter position

Index sets

$$
\begin{aligned}
& A \quad \operatorname{arcs} \\
& N \quad \operatorname{nodes} N=0,1,, m, m+1
\end{aligned}
$$


$T$ likely transmitter positions

Parameters

$m$ number of potential DF locations

$n$ number of DFs to be located

$r_{t}$ probability that a transmitter is actually positioned at $t$

$a\left(t, l_{i}, l_{j}\right)$ area of the quadrilateral (i.e., the surrogate measure) formed by error cones associated with DFs at locations $l_{i}$ and $l_{j}$ and an enemy transmitter at position $t$

Decision variables

$x_{i} j \quad 1$ if $\operatorname{arc}(i, j) \in A$ is selected, 0 otherwise.

The deterministic DF location problem $(\mathrm{P})$ can be stated as

\section{Model P:}

$$
\begin{aligned}
Z_{P}^{*}=\max & \sum_{(i, j) \in A} \sum_{t \in T} r_{t} a\left(t, l_{i}, l_{j}\right) x_{i j} \\
\text { s.t. } \quad & \sum_{j \in N} x_{i j}-\sum_{j \in N} x_{j i}= \begin{cases}1, & i=s \\
0 & i \neq s, e \\
-1 & i=e\end{cases} \\
& \sum_{(i, j) \in A} x_{i j}=n+1 \\
& x_{i j} \in\{0,1\}, \quad(i, j) \in A
\end{aligned}
$$

The objective (1) is to maximize the sum of weighted surrogate measures with the goal of estimating the positions of enemy transmitters as accurately as possible where ever they are among the set $T$ of likely positions within the ETA. Constraints (2) represent shortest-path constraints. Equality (3) is a side (i.e., resource) constraint, which requires that exactly $n$ nodes will be selected from among potential DF locations (the corresponding $n+1$ arcs prescribed are also incident from the start, and to the end, nodes). Constraints (4) require all decision variables to be binary. Because the network is acyclic, this problem can be solved as a minimization problem after multiplying the objective function by -1 . In this paper, we extend model $\mathrm{P}$, incorporating uncertainty associated with the enemy detecting DF locations.

Throughout this paper, we assume that likely transmitter positions within the ETA and potential DF locations within the friendly area can be identified by the DF system operator. Positions are likely and locations are potential if they are physically feasible and meet military requirements. Our models allow the probability of DF detection to be specified for each potential location so that the distance from the FLOT as well as natural concealments can be taken into consideration simply by assigning the appropriate probability. In our computational tests, we assume that the probability that a DF will be detected by the enemy depends on its location relative to the FLOT. That is, the probability that the enemy will detect a DF decreases as the distance from the FLOT increases.

\section{Models and Solution Methods}

This section presents our models in three sections. The first section describes two models based on expected values and describes the solution method we propose; the second formulates two CVaR models; and the third gives a scaled version of each type of model. Collectively, these models provide the battlefield commander the capability to deal with a variety of battlefield situation. 


\subsection{Expected Value Models}

In this section, we present two formulations of the stochastic DF location problem, each of which uses the surrogate measure of Kim and Wilhelm [8], and propose efficient solution methods.

\subsubsection{Expected value constraint model}

We propose an additional side constraint in problem $\mathrm{P}$ to incorporate the (perhaps) unique probability that a DF will be detected at each potential location, assuming that these probabilities can be estimated by the military commander or system operator. We now define some additional notation.

\section{Parameters}

$p_{l} \quad$ probability that a DF at location 1 will remain undetected over the planning horizon

$d$ minimum desirable expected number of DFs that remain undetected over the planning horizon.

DF deployment model P can be augmented by constraint (6), which requires that an expected number of at least $\mathrm{d}$ DFs remains undetected over the planning horizon:

\section{Model E1:}

$$
\begin{aligned}
Z_{E 1}^{*}=\max & \sum_{(i, j) \in A} \sum_{t \in T} r_{t} a\left(t, l_{i}, l_{j}\right) x_{i j} \\
\text { s.t. } \quad & (2)-(4) \\
& \sum_{(i, j) \in A} \frac{1}{2}\left(p_{i}+p_{j}\right) x_{i j} \geq d
\end{aligned}
$$

Given decision vector $\mathrm{x}$, the left-hand-side of constraint (6) computes the expected number of DFs that remain undetected over the planning horizon. Here, the term $\left(p_{i}+p_{j}\right)$ is multiplied by $1 / 2$ because, on a feasible path (i.e., in Figure 3), each node representing a DF location is counted twice, once at the head of an incoming arc and once at the tail of an outgoing arc.

Kim and Wilhelm [8] modified the label-setting algorithm (LSA) of Desrochers and Soumis [4] to solve problem P. Each label on a node represents a different path from the start node to that node and consists of a pair of numbers representing the cost and the weight of the corresponding path. Dumitrescu and Boland [5] used LSA to solve the weight-constrained shortest path problem (WCSPP) with one " $\leq$ " side constraint. Zhu and Wilhelm [16] extended the LSA of Dumitrescu and Boland [5] to solve the multiple resource constrained shortest path problem (MRCSP), which has more than one " $\leq$ " side constraints.

Note that model E1 has two side constraints: one " $\geq$ " constraint (6) and one equality (3). To deal with these constraints, we modify certain definitions used by Dumitrescu and Boland [5]. We let $L_{i}$ denote the index set of labels associated with node $i$; $E_{i}$, the index set of labels associated with node $i$; and $T_{i} \subseteq E_{i}$, the index subset of labels associated with node $i$ that have been treated. For each $k \in E_{i}$, let $P_{i}^{k}$ denote the path from the start node to node $i ; W_{i,=}^{k}\left(W_{i, \geq}^{k}\right)$, its cumulative weight corresponding to the " $=$ " (" $\geq$ ") constraint; and $C_{i}^{k}$, its cumulative objective function value. We use the following definitions.

Definition 1. For two different paths, $P_{i}^{k}$ and $P_{i}^{l}$, into node $i$, label $\left(W_{i,=}^{k}, W_{i, \geq}^{k}, C_{i}^{k}\right)$ dominates $\left(W_{i,=}^{l}, W_{i, \geq}^{l}, C_{i}^{l}\right)$ if and only if $W_{i,=}^{k} \geq W_{i,=}^{l}, W_{i, \geq}^{k} \geq W_{i, \geq}^{l}, C_{i}^{k} \leq C_{i}^{l}$ and at least one of the inequalities holds at strict inequality. Definition 2. If label $\left(W_{i,=}^{k}, W_{i, \geq}^{k}, C_{i}^{k}\right)$ is not dominated by any other label at node $i$, the label is said to be efficient and the corresponding path $P_{i}^{k}$ is said to be efficient.

With these definitions, our modified LSA identifies all efficient labels for every node so that a solution can be found by processing the end node. We now detail our modified LSA for model E1. 
STEP 0. Set $L_{0}=\{(0,0,0)\}, E_{0}=\{1\}$ and $L_{i}=E_{i}=\emptyset$ for all $i \in N \backslash\{0\}$

Set $T_{i}=\emptyset$ for each $i \in N$ and $U=\infty$

STEP 1 . for $i=0,1, \ldots, m$

while $E_{i} \backslash T_{i} \neq \emptyset$

$$
\text { choose } \mathrm{k}=\arg \min _{k^{\prime} \in E_{i} \backslash T_{i}}\left(W_{i,=}^{k^{\prime}}\right)
$$

$$
\begin{array}{r}
\text { for all }(i, j) \in A \text { with } W_{i=}^{k}+w_{i j=} \leq n+1 \text { and } C_{i}^{k}+c_{i j} \leq U \\
\quad \text { if }\left(W_{i,=}^{k}+w_{i j=}, W_{i,>}^{k}+w_{i j \geq}, C_{i}^{k}+c_{i j}\right) \text { is not dominated }
\end{array}
$$
by $\left(W_{i,=}^{l}, W_{i, \geq}^{l}, C_{i}^{l}\right)$ for any $l \in E_{j}$

then set $L_{j}=L_{j} \cup\left\{\left(W_{i,=}^{k}+w_{i j=}, W_{i, \geqslant}^{k}+w_{i j \geqslant}, C_{i}^{k}+c_{i j}\right)\right\}$ and add a corresponding index to $E_{j}$

$$
\begin{aligned}
& \text { if } W_{i,=}^{k}+w_{i j=}=n+1, W_{i, \geqslant}^{k}+w_{i j \geqslant} \geq d \text { and } C_{i}^{k}+c_{i j}<U \\
& \text { then } U=C_{i}^{k}+c_{i j} \\
& \text { set } T_{i}=T_{i} \cup\{k\}
\end{aligned}
$$

STEP 0 initializes labels $L_{0}$ and $E_{0}$ for the start node, assuming its index number is 1 (i.e., $E_{0}=\{1\}$ ). STEP 1 (i) selects a label associated with node $\mathrm{i}$ that is minimal with respect to $W_{i,=}^{k}$. STEP 1 (ii) treats the selected label, dealing with arc $(i, j)$, which is incident from node $i$ with $W_{i,=}^{k}+w_{i j=} \leq n+1$ and $C_{i}^{k}+c_{i j} \leq U$. STEP 1 (iii) adds a new label at the selected node $j$ only if it is not dominated by any other label on node $j$. If the $W_{=}$and $W_{\geq}$ associated with the added label are feasible with respect to side constraints (3) and (6), respectively, and, further, associated $\operatorname{cost} C$ is less than the current objective function value, STEP 1 (iv) updates upper bound $U$. Unlike other label-setting algorithms $[4,16]$, our algorithm can update the upper bound without preprocessing, because the structure of our network assures that the equality constraint is satisfied at each node that is directly connected to the end node.

\subsubsection{Expected value objective model}

Model E1 includes constraint (6), which may lead to infeasibility if the right-hand side value d is large. Thus, we also propose model E2, which incorporates the expected number of undetected DFs in the objective function, allowing the commander to locate DFs so that the optimal number of DFs remain undetected over the planning horizon. Kim and Wilhelm [8] demonstrated that model P locates DFs as closely as possible to FLOT to enhance the accuracy with which transmitter positions can be estimated. However, as DFs approach the FLOT, the risk posed by the enemy may increase as the likelihood of DF detection may increase. Therefore, this model can evaluate the trade-off between position-estimation accuracy and the number of DFs that remain undetected over the planning horizon.

Model E2:

$$
\begin{aligned}
Z_{E 2}^{*}=\max & \sum_{(i, j) \in A} \sum_{t \in T} r_{t} a\left(t, l_{i}, l_{j}\right) x_{i j}+\sum_{(i, j) \in A} \frac{1}{2}\left(p_{i}+p_{j}\right) x_{i j} \\
\text { s.t. } & (2)-(4)
\end{aligned}
$$

Objective function (7) maximizes the sum of the surrogate measures and the expected number of undetected DFs. Model E2 incorporates constraints (2)-(4) as does model P. Thus, we propose that it be solved using the modified LSA discussed by Kim and Wilhelm [8].

\subsection{CVaR models}

In actual situations, it may not be possible to estimate probability $p_{i}$ accurately. To compensate, we formulate robust DF location models based on the CVaR measure. One way to cope with risk caused by uncertainty is to plan for the worst-case scenario. 
Value at risk (VaR) is a measure of risk that is a widely used in finance; it prescribes the maximum loss by specifying a confidence level (CL). Although $\mathrm{VaR}$ is a popular measure of risk, it has shortcomings in optimization problems; in particular, it is not a convex measure. CVaR, an alternative measure of risk that satisfies convexity (Artzner et al, [3]), has been applied successfully in a number of areas, including investment portfolios (Rockafellar et al, [12]), target assignment to weapons (Krokhmal et al, [9]), and hazardous material transportation (Toumazis et al, [14]). In many military applications, a decision must not only be effective on average, but also safe enough under a wide range of possible scenarios. In this sense, CVaR can be an appropriate measure. Formal definitions of $\mathrm{VaR}$ and $\mathrm{CVaR}$ can be found in $[12,15]$.

\subsubsection{CVaR constraint model}

We now incorporate function $\mathrm{CVaR}$ in the $\mathrm{DF}$ location problem, using the following additional notation. Scenarios may be constructed based on historical data of detection in different environments, simulated data, commanders assessment, or experts views (e.g., Delphi method).

Index

$s$ scenario

Index set

$S$ scenarios

Parameters

$p_{l s} \quad$ probability that a DF at location $l$ will remain undetected over the planning horizon under enemy attack scenario $s$

$P_{s} \quad$ probability that attack scenario $\mathrm{s}$ is realized

VaR and CVaR were proposed to cope with risk in the financial industry and are widely used to measure the risk associated with portfolios. In general, risk management in this industry aims to reduce, if not prevent, high losses caused by bad decisions. A loss function, which depends on decisions and vectors of uncertain parameters, is used to quantify the loss. Because we consider the expected number of undetected DFs as a loss, we multiply this number by -1 so that the loss is actually beneficial. Letting $g(\mathbf{x}, \mathbf{s})$ be the loss function related to decision $\mathbf{x}$ under scenario $s$, we have

$$
g(\mathbf{x}, s)=\sum_{(i, j) \in A} \frac{1}{2}\left(p_{i s}+p_{j s}\right) x_{i j}
$$

so that the corresponding CVaR measure (or $\beta-\mathrm{CVaR}$ ) is

$$
\alpha+\frac{1}{1-\beta} \sum_{s \in S} P_{s}[g(\mathbf{x}, s)-\alpha]^{+}, \quad \alpha \in R .
$$

By restricting CVaR $\leq-d$, requiring the expected number of undetected DFs in the $(1-\beta) 100 \%$ worst cases to be greater than or equal to $d$, we obtain the following CVaR model :

$$
\begin{aligned}
Z_{C 1}^{*}=\max & \sum_{(i, j) \in A} \sum_{t \in T} r_{t} a\left(t, l_{i}, l_{j}\right) x_{i j} \\
\text { s.t. } \quad & (2)-(4) \\
& \alpha+\frac{1}{1-\beta} \sum_{s \in S} P_{s}[g(\mathbf{x}, s)-\alpha]^{+} \leq-d \\
& \alpha \in R .
\end{aligned}
$$


Constraint (9) imposes $\mathrm{CVaR} \leq-d$. Constraint (10) indicates that $\alpha$ is not sign restricted. By introducing an auxiliary, nonnegative variable associated with scenario $s \in S, z_{s}$, constraint (9) can be linearized as follows:

$$
\begin{array}{rl}
\alpha+\frac{1}{1-\beta} \sum_{s \in S} P_{s} z_{s} & \leq-d \\
g(\mathbf{x}, s)-\alpha-z_{s} \leq 0 & s \in S \\
z_{s} \geq 0 & s \in S
\end{array}
$$

\subsubsection{CVaR objective model}

Constraint (9) forces the expected number of undetected DFs to be more than $d$, even in the $(1-\beta) 100 \%$ worst cases. This implies that model $\mathrm{C} 1$ is quite conservative and, therefore, more likely to be infeasible than model E1, assuming that both models are applied to the same instance. Thus, we also propose a model that incorporates $\mathrm{CVaR}$ along with the surrogate measure in the objective function so that trade-offs between the accuracy with which enemy transmitter positions are estimated and the risk of DF detection can be assessed as with (7).

Model C2:

$$
\begin{aligned}
Z_{C 2}^{*}=\max & \sum_{(i, j) \in A} \sum_{t \in T} r_{t} a\left(t, l_{i}, l_{j}\right) x_{i j}-\left(\alpha+\frac{1}{1-\beta} \sum_{s \in S} P_{s} z_{s}\right) \\
\text { s.t. } & (2)-(4),(10),(12),(13)
\end{aligned}
$$

Objective function (14) maximizes the sum of the surrogate and revised CVaR measures. Because CVaR is the conditional expectation of loss, we include CCVaR in the maximizing objective function with the goal of minimizing CVaR.

\subsection{Scaling measures}

The objective functions of models E2 and C2 ((7) and (14), respectively) each add two measures, one of which may be expressed in units that are much larger than the other, artificially ascribing a higher level of importance to that measure. To rectify this imbalance so that meaningful trade-off analysis of the accuracy of transmitter-position estimation accuracy vs the expected number of DFs that remain undetected can be undertaken, we scale each summation.

We scale the first summation in (7) by solving model $\mathrm{P}$ (1)-(4) to obtain $Z_{P}^{*}$ and solve $\max \left\{\sum_{(i, j) \in A} \frac{1}{2}\left(p_{i}+p_{j}\right) x_{i j}:(2),(3), \operatorname{and}(4)\right\}$ to obtain $p_{\max }$. The resulting, scaled version of E2, SE2, is

$$
\begin{aligned}
Z_{S E 2}^{*}=\max & \frac{w}{Z_{P}^{*}} \sum_{(i, j) \in A} \sum_{t \in T} r_{t} a\left(t, l_{i}, l_{j}\right) x_{i j}+\frac{(1-w)}{p_{\max }} \sum_{(i, j) \in A} \frac{1}{2}\left(p_{i}+p_{j}\right) x_{i j} \\
\text { s.t. } & (2)-(4)
\end{aligned}
$$

Scaling puts both measures on a common scale between 0 and 1 . We also weight the terms with $w$ and $(1-w)$, respectively, where $0 \leq w \leq 1$. Similarly, we scale the summations in (14) using $Z_{P}^{*}$ and obtain $q_{m} a x$ by solving $\max \left\{-\left(\alpha+\frac{1}{1-\beta} \sum_{s \in S} P_{s} z_{s}\right):(2),(3),(4),(10),(12)\right.$, and (13) $\}$. The scaled, weighted version of C2, SC2, is

$$
\begin{aligned}
Z_{S E 2}^{*}=\max & \frac{w}{Z_{P}^{*}} \sum_{(i, j) \in A} \sum_{t \in T} r_{t} a\left(t, l_{i}, l_{j}\right) x_{i j}-\frac{(1-w)}{q_{m} a x}\left(\alpha+\frac{1}{1-\beta} \sum_{s \in S} P_{s} z_{s}\right) \\
\text { s.t. } & (2)-(4),(10),(12),(13)
\end{aligned}
$$


We test three values of $w(w=0.25,0.5$, or 0.75$)$ to weigh the accuracy of position estimation and DF detection measures in objective functions (15) and (16) in a sensitivity analysis.

\section{Computational evaluation}

In this section, we compare solutions obtained computationally from models P, E1, E2, SE2, C1, C2, and SC2. We implement the algorithms for solving P, E1, E2, and SE2 in C/C++ and use CPLEX to solve C1, C2, and SC2, performing all computations on a Dell PC running a $2.67 \mathrm{GHz}$ CPU with Windows 7 and 2 GB RAM.

\subsection{Test instances}

We use test instances generated by Kim and Wilhelm [8]. Because there may be a large number of likely positions and potential locations, they placed a grid over a military map, defining cells of specified size and considering the centers of certain cells as likely transmitter positions or potential DF locations. Their tests involved three factors, each with either two or three levels: the shapes of FLOTs (convex or concave), distributions of likely transmitter positions (left-skewed, centered, and right-skewed), and sizes of grids $\left(1 \times 1 \mathrm{~km}^{2}, .5 \times .5 \mathrm{~km}^{2}\right.$, and $\left..25 \times .25 \mathrm{~km}^{2}\right)$.

We select convex and concave shapes of the FLOT to study the effects of different geometries, not because of their prevalence on actual battlefields. We use instances with large numbers of potential DF locations (i.e., instances with $.5 \times .5 \mathrm{~km}^{2}$ or $.25 \times .25 \mathrm{~km}^{2}$ cells). Each instance has 1, 2, or 3 groups of cells (G1, G2, G3) representing likely transmitter positions. The rows of Table 1 specify these 12 test instances; columns give, respectively, instance number; cell size; number of DF locations (m) and transmitter positions $(|T|)$; and the $r_{t}$, which we assume is the same for each cell in each group.

Table 1. Performance of Random Variable Partition

\begin{tabular}{|c|c|c|c|c|c|c|c|c|c|c|c|c|c|}
\hline \multirow{2}{*}{ Inst. } & $\begin{array}{c}\text { Cell Size } \\
\left(\mathrm{km}^{2}\right)\end{array}$ & \multirow{2}{*}{$\mathrm{m}$} & $|T|$ & \multicolumn{3}{|c|}{$r_{t}$} & \multirow{2}{*}{ Inst. } & $\begin{array}{c}\text { Cell Size } \\
\left(\mathrm{km}^{2}\right)\end{array}$ & $\mathrm{m}$ & $|T|$ & \multicolumn{3}{|c|}{$r_{t}$} \\
\cline { 8 - 14 } & & & $\mathrm{G} 1$ & $\mathrm{G} 2$ & $\mathrm{G} 3$ & & & $\mathrm{G} 1$ & $\mathrm{G} 2$ & $\mathrm{G} 3$ \\
\hline 1 & $.25 \times .25$ & 454 & 731 & 0.4 & 0.6 & & 7 & $.5 \times .5$ & 114 & 200 & 0.4 & 0.6 & \\
2 & $.25 \times .25$ & 483 & 642 & 0.8 & & & 8 & $.5 \times .5$ & 124 & 161 & 0.8 & & \\
3 & $.25 \times .25$ & 483 & 836 & 0.4 & 0.7 & 0.5 & 9 & $.5 \times .5$ & 118 & 198 & 0.4 & 0.7 & 0.5 \\
4 & $.25 \times .25$ & 487 & 690 & 0.6 & 0.4 & & 10 & $.5 \times .5$ & 137 & 162 & 0.6 & 0.4 & \\
5 & $.25 \times .25$ & 500 & 570 & 0.8 & & & 11 & $.5 \times .5$ & 126 & 142 & 0.8 & & \\
6 & $.25 \times .25$ & 504 & 744 & 0.8 & 0.6 & 0.4 & 12 & $.5 \times .5$ & 121 & 187 & 0.8 & 0.6 & 0.4 \\
\hline
\end{tabular}

Two DFs can estimate the position of a transmitter but U.S. Army FM 34-40-9 [1] recommends that at least three DFs be used to enhance position-estimation accuracy. Thus, we consider systems with either 4 or 5 DFs, requiring the minimum desirable expected number of undetected DFs to be $d=3$. For each instance, we divide the area on the friendly side of the FLOT into three regions $\left(R_{1}, R_{2}\right.$, and $\left.R_{3}\right)$, each of $1 \mathrm{~km}$ width, and assume that all cells in a given region have the same probability of DF detection. Figure 4 depicts these regions for both convex and concave FLOTs.

For CVaR models $\mathrm{C} 1$ and $\mathrm{SC} 2$, we set $\mathrm{CL} \beta=0.9$ and use 5 probability scenarios $p_{l s}: s \in S=\{1, \ldots, 5\}$. We assume that all scenarios are equally probable (i.e., $P_{s}=P_{q}, s, q \in S, s \neq q$ ) and that the probability of detecting a $\mathrm{DF}$ at a location is determined by its proximity to FLOT. Table 2 displays the values we assume for the probability that a DF will remain undetected in each cell in each region under each scenario. Expected value models E1 and SE2 use the probabilities that a DF will remain undetected given in the last column; each of these values is averaged over the five scenarios. 


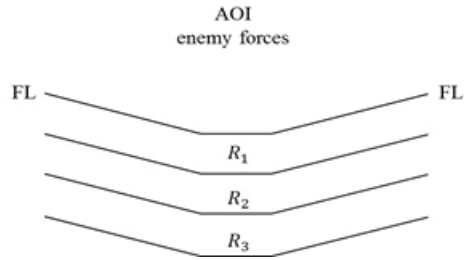

(a) Convex FLOT

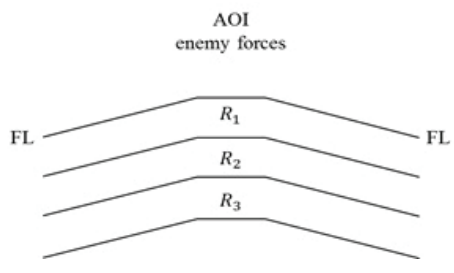

(b) Concave FLOT

Figure 4. Probabilities of DF detection by the enemy

Table 2. Probability that a DF will remain undetected in each region under each scenario

\begin{tabular}{|c|c|c|c|c|c|c|}
\hline Region $\backslash$ Scenario & Scenario 1 & Scenario 2 & Scenario 3 & Scenario 4 & Scenario 5 & Average \\
\hline$R_{1}$ & 0.55 & 0.60 & 0.65 & 0.70 & 0.75 & 0.65 \\
\hline$R_{2}$ & 0.65 & 0.70 & 0.75 & 0.80 & 0.85 & 0.75 \\
\hline$R_{3}$ & 0.75 & 0.80 & 0.85 & 0.90 & 0.95 & 0.85 \\
\hline
\end{tabular}

\subsection{Computational results}

We first compare the objective function values and DF locations prescribed by three models P, E1 and C1. Recall that models, $\mathrm{P}, \mathrm{E} 1$, and $\mathrm{C} 1$ have the same objective function.

Table 3. Results of models P, E1, and C1 $\left(.25 \times .25 \mathrm{~km}^{2}\right)$ cells

\begin{tabular}{|c|c|c|c|c|c|c|c|}
\hline$(1)$ & (2) & (3) & (4) & $(5)$ & (6) & (7) & (8) \\
\hline \multirow{2}{*}{ \# of DFs } & \multirow{2}{*}{ Inst. } & \multicolumn{2}{|c|}{ Model P } & \multicolumn{2}{|c|}{ Model E1 } & \multicolumn{2}{|c|}{ Model C1 } \\
\hline & & $Z_{P}^{*}$ & Locations & $Z_{E 1}^{*}$ & Locations & $Z_{C 1}^{*}$ & Locations \\
\hline \multirow{6}{*}{4 DFs } & 1 & 201.30 & $1,1,1,1$ & 185.93 & $2,1,3,1$ & 142.66 & $2,3,3,3$ \\
\hline & 2 & 282.01 & $1,1,1,1$ & 254.41 & $2,1,2,1$ & 213.99 & $3,3,3,3$ \\
\hline & 3 & 282.26 & $1,1,1,1$ & 248.50 & $3,3,1,1$ & 208.38 & $2,3,3,3$ \\
\hline & 4 & 204.97 & $1,1,1,1$ & 185.88 & $2,1,2,2$ & 150.35 & $3,3,3,3$ \\
\hline & 5 & 176.52 & $1,1,1,1$ & 161.73 & $2,1,2,2$ & 130.70 & $3,3,3,3$ \\
\hline & 6 & 221.05 & $1,1,1,1$ & 197.24 & $2,2,1,2$ & 165.33 & $3,3,3,3$ \\
\hline \multirow{6}{*}{$5 \mathrm{DFs}$} & 1 & 210.67 & $1,1,1,1,1$ & 210.67 & $2,1,1,1,1$ & 201.38 & $2,1,1,3,1$ \\
\hline & 2 & 295.08 & $1,1,1,1,1$ & 295.08 & $1,1,1,1,1$ & 279.61 & $3,2,1,1,1$ \\
\hline & 3 & 293.04 & $1,1,1,1,1$ & 293.04 & $1,1,1,1,1$ & 293.04 & $1,1,1,1,1$ \\
\hline & 4 & 216.73 & $1,1,1,1,1$ & 216.73 & $1,1,1,1,1$ & 203.82 & $2,1,1,1,3$ \\
\hline & 5 & 180.85 & $1,1,1,1,1$ & 180.85 & $1,1,1,1,1$ & 172.52 & $1,1,1,2,3$ \\
\hline & 6 & 239.69 & $1,1,1,1,1$ & 239.69 & $1,1,1,1,1$ & 226.97 & $3,1,2,1,1$ \\
\hline
\end{tabular}

Table 3 displays results of tests on instances with $.25 \times .25 \mathrm{~km}^{2}$ cells. Rows 4-9 (10-15) give objective function values and DF locations for 4 DFs (5 DFs). Columns 1 and 2 give the number of DFs to be located and the instance number, respectively. Columns 3,5, and 7 display the objective function values for each of the three models. Numbers in columns 4,6 , and 8 give the indices of the region (i.e., $R_{i}, i=1,2,3$ ) in which each of the 4 , respectively 5 , DFs is located.

We first compare objective function values for 4 DFs. $Z_{E 1}^{*}$ and $Z_{C 1}^{*}$ are less, respectively, by $12.3 \%$ and $24.7 \%$, on average, than $Z_{P}^{*}$. These decreases in the objective function values result from deploying DFs further away from 
the FLOT, increasing the probability that a DF will remain undetected at the expense of reducing the accuracy with which transmitter positions can be estimated. Tests conducted by Kim and Wilhelm [8] showed that DFs deployed in cells adjacent to the FLOT enhance transmitter-position estimation accuracy. As shown in Table 3, models E1 and C1 locate some or all DFs away from the FLOT to satisfy constraints (6) and (9), respectively, increasing the expected number of DFs that remain undetected. Results indicate that locations prescribed by model $\mathrm{P}$ can estimate transmitter position most accurately and those prescribed by model $\mathrm{C} 1$ have the most desirable levels of detection.

With $5 \mathrm{DFs}$, objective function values $Z_{P}^{*}, Z_{E 1}^{*}$ and $Z_{C 1}^{*}$ are nearly the same. For each instance, model E1 gives the same objective function value that model P gives. $Z_{C 1}^{*}$ is $3.81 \%$ less than $Z_{P}^{*}$, on average. Columns 4,6 , and 8 show that these three models locate most DFs in region $R_{1}$, which has the highest DF-detection probability. This seems counter-intuitive but results because it is more likely for 5 DFs to satisfy the requirement that at least $d=3$ DFs remain undetected than 4 DFs, even if DFs at forward locations have higher probability of detection. Results also show that model $\mathrm{C} 1$ prescribes the most conservative (i.e., risk adverse) locations with some DFs in regions $R_{2}$ and $R_{3}$.

Because of space limitations, we show results graphically for only instance 1, which is, however, typical. Figure 5 depicts the 4 DF locations prescribed by each of the three models: P, E1 and C1. Groups of Cells (rectangleshaped) above the FLOT represent likely transmitter positions and white and black dots below the FLOT denote the potential and selected DF locations, respectively.

Figure 5 (b) shows that model E1 does not deploy all DFs in cells adjacent to FLOT; it locates 2 DFs in region $R_{1}, 1 \mathrm{DF}$ in region $R_{2}$, and $1 \mathrm{DF}$ in region $R_{3}$. DFs in regions $R_{2}$ and $R_{3}$ have high enough probabilities of a DF remaining undetected to satisfy constraint (6). However, transmitter position-estimation accuracy decreases, resulting in a decreased objective function value in comparison with model P. Model $\mathrm{C} 1$, which incorporates the CVaR constraint, locates all DFs in region $R_{3}$ where DF detection probability is the lowest. This shows that the CVaR-constrained model prescribes DF locations that estimate enemy positions with lesser accuracy but the most risk adverse set of DF locations.

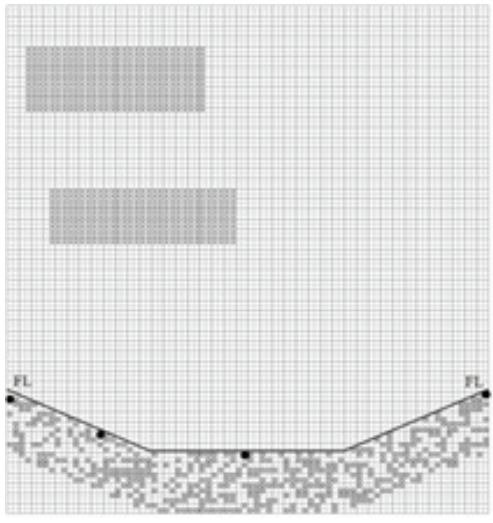

(a) Model P

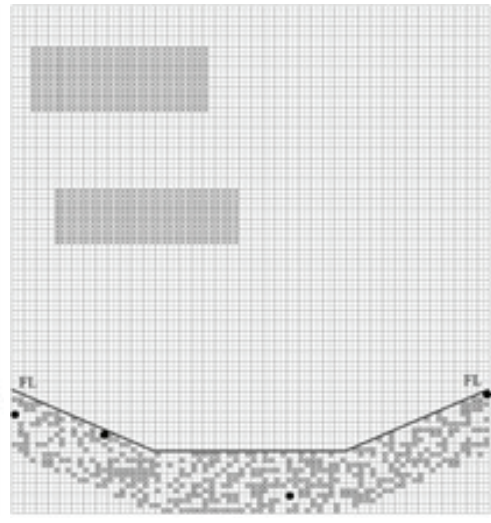

(b) Model E1

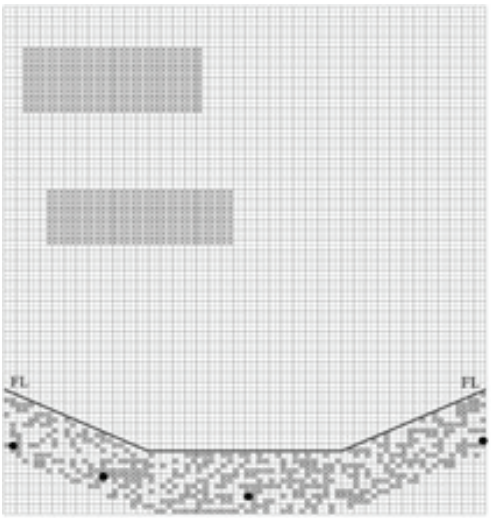

(c) Model C1

Figure 5. DF locations for models P, E1, and C1

We now analyze the sensitivity of weight $w$, using the results of weighted, scaled models SE2 and SC2. Table 4 displays results for instances with 4 DFs (We have the similar result from $5 \mathrm{DFs}$ ). Columns 1 and 2 provide the number of DFs to be located and the instance number, respectively. Column 3 gives, the weight assigned to transmitter-positon estimation accuracy each instance. Columns 4 and 6 (5 and 7) display the objective function values (the region in which each DF is located) for each of the two scaled models.

Objective function values $Z_{S E 2}^{*}$ and $Z_{S C 2}^{*}$ are nearly the same, as shown in Table 4. Results show that DF locations depend on $\mathrm{w}$ values. As $\mathrm{w}$ increases (i.e., transmitter position estimation accuracy is weighted more heavily than the DF- detection measure), DFs tend to approach FLOT to increase position-estimation accuracy. When $w=0.50$, however, both models tend to deploy some DFs away from FLOT and some DFs close to FLOT. 
Table 4. Results of models SE2 and SC2 $\left(.25 \times .25 \mathrm{~km}^{2}\right)$ cells

\begin{tabular}{|c|c|c|c|c|c|c|}
\hline$(1)$ & (2) & (3) & $(4)$ & (5) & (6) & (7) \\
\hline \multirow{2}{*}{ \# of DFs } & \multirow{2}{*}{ Inst. } & \multirow{2}{*}{$w$} & \multicolumn{2}{|c|}{ Model SE2 } & \multicolumn{2}{|c|}{ Model SC2 } \\
\hline & & & $Z_{S E 2}^{*}$ & Locations & $Z_{S C 2}^{*}$ & Locations \\
\hline \multirow{18}{*}{$4 \mathrm{DFs}$} & \multirow{3}{*}{1} & 0.25 & 0.93 & $3,3,3,3$ & 0.93 & $3,3,3,3$ \\
\hline & & 0.50 & 0.90 & $2,3,1,1$ & 0.88 & $2,1,1,1$ \\
\hline & & 0.75 & 0.95 & $2,3,1,1$ & 0.94 & $2,1,1,1$ \\
\hline & \multirow{3}{*}{2} & 0.25 & 0.94 & $3,3,3,3$ & 0.94 & $3,3,3,3$ \\
\hline & & 0.50 & 0.89 & $3,1,1,1$ & 0.88 & $3,1,3,1$ \\
\hline & & 0.75 & 0.94 & $1,1,1,1$ & 0.93 & $1,1,1,1$ \\
\hline & \multirow{3}{*}{3} & 0.25 & 0.91 & $3,3,3,1$ & 0.98 & $2,1,1,1$ \\
\hline & & 0.50 & 0.89 & $3,1,1,1$ & 0.97 & $1,1,1,1$ \\
\hline & & 0.75 & 0.94 & $1,1,1,1$ & 0.98 & $1,1,1,1$ \\
\hline & \multirow{3}{*}{4} & 0.25 & 0.93 & $3,3,3,3$ & 0.93 & $3,3,3,3$ \\
\hline & & 0.50 & 0.88 & $3,3,1,1$ & 0.88 & $3,3,1,1$ \\
\hline & & 0.75 & 0.94 & $1,1,1,1$ & 0.93 & $1,1,1,1$ \\
\hline & \multirow{3}{*}{5} & 0.25 & 0.94 & $3,3,3,3$ & 0.94 & $3,3,3,3$ \\
\hline & & 0.50 & 0.89 & $2,3,1,1$ & 0.88 & $3,2,2,2$ \\
\hline & & 0.75 & 0.94 & $2,3,1,1$ & 0.94 & $3,1,2,3$ \\
\hline & \multirow{3}{*}{6} & 0.25 & 0.93 & $3,3,3,3$ & 0.94 & $2,3,3,3$ \\
\hline & & 0.50 & 0.89 & $3,1,1,1$ & 0.89 & $2,3,3,3$ \\
\hline & & 0.75 & 0.94 & $1,1,1,1$ & 0.93 & $3,1,3,1$ \\
\hline
\end{tabular}

These results show that there is a trade-off between the accuracy of position estimation and the DF detection measure. Using instances with cell sizes of $.5 \times .5 \mathrm{~km}^{2}$, we obtain results that are similar to those discussed in detail above, which have cell size $.25 \times .25 \mathrm{~km}^{2}$. Thus, we omit the detailed explanation.

Finally, we compare the run times required by our modified label-setting algorithm and CPLEX 12.1.0. We only test instances with the $.25 \times .25 \mathrm{~km}^{2}$ cells because they require longer run times than those with $.5 \times .5 \mathrm{~km}^{2}$ cells. Table 5 displays results. In the table, columns 3,5, and 7 provide the runtimes required by the label-setting algorithm in application to model P, E1, and E2, respectively. Columns 4, 6, and 8 give the CPLEX runtimes that correspond to those in columns 3,5, and 7, respectively.

Results show that the LSA outperforms CPLEX relative to runtimes. Results also show that LSA runtime increases with the number of constraints (see P vs. E1 and E1 vs. E2). This relationship is due to the fact that LSA is a pseudo-polynomial time algorithm: its runtime depends on the number of constraints and the magnitude of right-hand-side values (Desrochers et al, [4]; Zhu et al, [16]).

\section{Conclusions and recommendations for future research}

On the battlefield, both friend and foe attempt to seek and destroy the other side. This is the first study to incorporate the possibility of DF detection by the enemy in the DF location problem, which holds the goal of maximizing the accuracy of transmitter-position estimation while hedging against the risk of DF detection by the enemy.

Our computational tests identify trade-offs between the accuracy with which enemy transmitter positions are estimated and the risk of DF detection. These tradeoffs, which have not been identified by previous research, provide useful guidelines to support a commanders decisions on the battlefield. Our models reveal insights that can help a field commander in locating DFs whether optimization models are used or not. These insights complement the information provided by the FM 34-40-9 (Army [1]), a recognized source used by militaries around the world. 
Table 5. Comparison of runtimes (secs.) $\left(.25 \times .25 \mathrm{~km}^{2}\right)$ cells

\begin{tabular}{|c|c|c|c|c|c|c|c|}
\hline (1) & $(2)$ & $(3)$ & $(4)$ & $(5)$ & $(6)$ & $(7)$ & $(8)$ \\
\hline \multirow{4}{*}{ \# of DFs } & \multirow{2}{*}{ Inst. } & \multicolumn{2}{|c|}{ Model P } & \multicolumn{2}{c|}{ Model E1 } & \multicolumn{2}{c|}{ Model E2 } \\
\cline { 2 - 7 } & & LSA & CPLEX & LSA & CPLEX & LSA & CPLEX \\
\hline \multirow{5}{*}{ 4 DFs } & 1 & 0.00 & 1.03 & 0.06 & 3.29 & 0.00 & 1.95 \\
& 2 & 0.00 & 1.11 & 0.06 & 6.63 & 0.02 & 2.78 \\
& 3 & 0.00 & 1.19 & 0.06 & 3.35 & 0.00 & 2.25 \\
& 4 & 0.01 & 1.31 & 0.06 & 2.79 & 0.01 & 3.31 \\
& 5 & 0.00 & 1.40 & 0.07 & 3.11 & 0.01 & 3.18 \\
& 6 & 0.02 & 2.32 & 0.06 & 5.48 & 0.02 & 2.46 \\
& Avg. & 0.005 & 1.727 & 0.061 & 4.108 & 0.01 & 2.655 \\
\hline \multirow{5}{*}{ 5 DFs } & 7 & 0.00 & 0.97 & 0.06 & 4.88 & 0.00 & 2.29 \\
& 8 & 0.02 & 1.11 & 0.07 & 4.51 & 0.00 & 2.84 \\
& 9 & 0.00 & 1.33 & 0.07 & 3.18 & 0.00 & 3.10 \\
& 10 & 0.00 & 1.28 & 0.07 & 3.73 & 0.02 & 2.95 \\
& 11 & 0.02 & 3.70 & 0.08 & 2.96 & 0.02 & 3.43 \\
& 12 & 0.02 & 2.93 & 0.08 & 7.92 & 0.00 & 2.96 \\
& Avg. & 0.01 & 1.887 & 0.071 & 4.530 & 0.007 & 2.928 \\
\hline
\end{tabular}

If 4 DFs are used, models E1 and C1 tend to locate some or all DFs far from the FLOT to reduce risk. In particular, model $\mathrm{C} 1$ deploys all DFs (except instances 1 and 3) in region $R_{3}$ which offers the best probability that a DF will remain undetected (i.e., it is a risk-adverse solution). However, model C1 prescribes DF locations that estimate enemy positions with lesser accuracy. Results also show that solutions are very different from each other but objective function values are not, which indicates that the surrogate measure can be used to prescribe optimal locations but that solutions can give similar objective function values.

If $5 \mathrm{DFs}$ are used, models $\mathrm{P}$ and $\mathrm{E} 1$ deploy most DFs in region $R_{1}$; even though the probability that a DF in that region will be detected by the enemy is high compared with other regions, the expected number of DFs that will remain undetected will satisfy constraint (6). A system with $5 \mathrm{DFs}$ improves the number of undetected DFs. If either 4 or $5 \mathrm{DFs}$ are used, model $\mathrm{C} 1$ locates some DFs in regions $R_{2}$ and $R_{3}$ to hedge against the $(1-\beta) \%$ worst cases.

Whether 4 or 5 DFs are deployed, Models E2 and C2 are sensitive to weight $w$. Both models with weight $w=0.75(w=0.25)$ locate most DFs in region $R_{1}\left(R_{3}\right)$. With weight $w=0.50$, both models locate DFs in regions $R_{1}, R_{2}$, and $R_{3}$ to balance the accuracy of estimating transmitter positions with the number of DFs that will remain undetected.

The paper offers options for the commander to select a model that best suits battlefield conditions. In particular, it is often the case that time is of the essence on the battlefield and the label-setting algorithm we propose can solve any of the E models very quickly, enabling a commander to cope with an imminent enemy attack.

A commander can use an $\mathrm{E}$ model if $\mathrm{s} / \mathrm{he}$ is most interested in the number of DFs that can be expected to remain undetected and a $\mathrm{C}$ model if $\mathrm{s} / \mathrm{he}$ is risk averse. One might expect an aggressive commander on the offensive to select an $\mathrm{E}$ model and one that is beleaguered or primarily on the defensive to select a $\mathrm{C}$ model. If the constraints in model E1 (C1) cannot be satisfied, the commander can resort to model E2 (C2), essentially to achieve the best number of undetected DFs possible under battlefield conditions. Including both criteria in the objective allows the commander to assess tradeoffs between the two (perhaps) conflicting goals. Finally, models SE2 and SC2 allow the commander to weight each of the two components of the objective function to reflect his/her personal assessment of battlefield conditions. Our models deploy DFs according to the shape of FLOT, a practice that Army FM 34-409 (Army [1]) recommends. Thus, our results also reveal insights that complement the manual, providing decision support for the field commander. 
Instead of dealing with risk by considering the expected number of DFs that remain undetected, future research could work with the probability that 2 or more DFs will remain undetected, corresponding to the fact that at least 2 DFs are needed to estimate a transmitter position. In this and other studies associated with DF location (Kim et al, [8]; Lee et al, [10]), authors assumed that the probability that a transmitter will actually be in a likely position is known. However, it may be hard to know this probability on a real battlefield. In addition, at least three DFs are required to estimate the position of a transmitter accurately. Therefore, future research could address DF location to maximize the expected number of likely transmitter positions that are covered by at least three DFs.

\section{REFERENCES}

1. U.S. Army,Field Manual 34-4-9, Direction finding operations, Headquarters, Department of the Army: Washington, 1991.

2. U.S. Army,Field Manual 101-5-1, Operational terms and graphics, Headquarters, Department of the Army: Washington, 1997.

3. P. Artzner, F. Delbaen, J.M. Eber and D. Heath, Coherent measures of risk, Mathematical Finance 9.3: 203-228, 1999.

4. M. Desrochers and F.A. Soumis, A generalized permanent labeling algorithm for the shortest path problem with time windows, INFOR 26: 193-214, 1988 .

5. I. Dumitrescu and N. Boland, Algorithms for the weight constrained shortest path problem, International Transactions in Operational Research 8.1: 15-29, 2001.

6. H.D. Kennedy and R.B. Woolsey, Direction-Finding Antennas and Systems, in Antenna Engineering Handbook, 3rd Edition, edited by R.C. Johnson, Ed, New York: McGraw-Hill, Chapter 39, 1993.

7. H.H. Jenkins, Small-aperture radio direction-finding, Artech house, Norwood, 1991

8. S.H. Kim and W.E. Wilhelm, On the optimal deployment of direction finders, IEEE Transactions on Automation Science and Engineering 11(2), 2014.

9. P. Krokhmal, R. Murphey, P. Pardolas, and S. Uryasev, Robust decision making: Addressing uncertainties in distributions, Cooperative Control:Models, Applications, and Algorithms:165-185, 2003.

10. H.J. Lee, Y.D. Kim, and S.B. Lee, A simulation-based heuristic algorithm for disposition of direction finders, Computers and Industrial Engineering 55(1): 134-149. 2008.

11. L. Jingwen and S.A. Quek, Locating a target from directional data, Naval Research Logistics 45(4): 353-364, 1998.

12. R.T. Rockafellar and S. Uryasev, Optimization of conditional value-at-risk, Journal of risk 2: 21-42, 2000.

13. R.G. Stansfield, Statistical theory of DF fixing. Electrical Engineers-Part IIIA: Radiocommunication, Journal of the Institution of 94(15): 762-770, 1947.

14. I. Toumazis, C. Kwon, and R. Batta, Value-at-Risk and Conditional Value-at-Risk Minimization for Hazardous Materials Routing, Handbook of OR/MS Models in Hazardous Materials Transportation. Springer. New York, 2013.

15. S. Uryasev, Conditional value-at-risk: Optimization algorithms and applications, Computational Intelligence for Financial Engineering, Proceedings of the IEEE/IAFE/INFORMS 2000 Conference on. IEEE, 2000.

16. X. Zhu and W.E. Wilhelm, A three-stage approach for the resource-constrained shortest path as a sub-problem in column generation, Computers and Operations Research 39(2): 164-178, 2012. 